\title{
Lentinan mitigates therarubicin-induced myelosuppression by activating bone marrow-derived macrophages in an MAPK/NF-кB-dependent manner
}

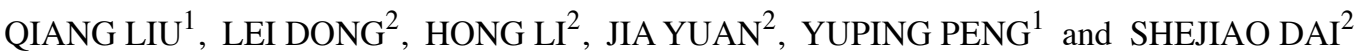 \\ Departments of ${ }^{1}$ Radiology and ${ }^{2}$ Gastroenterology, The Second Affiliated Hospital, \\ Medical School of Xi'an Jiaotong University, Xi'an, Shaanxi 710004, P.R. China
}

Received December 4, 2015; Accepted January 6, 2016

DOI: $10.3892 /$ or.2016.4769

\begin{abstract}
Bone marrow (BM) suppression (also known as myelosuppression) is the most common and most severe side-effect of therarubicin (THP) and thereby limits the clinical application of this anticancer agent. Lentinan (LNT), a glucan extracted from dried shiitake mushrooms (Lentinula edodes), exhibits a variety of pharmacological activities. The objectives of the present study were to determine the effect of LNT on the myelosuppression of THP-treated mice and to examine the pharmacological mechanism of these effects. In vivo experiments indicated that non-cytotoxic levels of LNT strongly increased blood myeloperoxidase (MPO) activity; improved $\mathrm{BM}$ structural injuries; increased the numbers of leukocytes and neutrophils in the blood and BM; elevated the blood levels of granulocyte colony-stimulating factor (G-CSF), granulocyte-macrophage colony-stimulating factor (GM-CSF) and macrophage colony-stimulating factor (M-CSF); and reduced the self-healing period in THP-treated mice. In vitro experiments indicated that LNT increased the viability of BM-derived macrophages (BMDMs) in a time- and dosedependent manner without toxic side-effects and markedly increased the release of G-CSF, GM-CSF and M-CSF by BMDMs. Further analyses revealed that LNT activated the $\mathrm{NF}-\kappa \mathrm{B}$ and MAPK signalling pathways and promoted the nuclear import of p65 and that BAY 11-7082 (a specific inhibitor of NF- $\mathrm{KB}$ ) suppressed the release of G-CSF, GM-CSF and M-CSF. Furthermore, we found that U0126, SB203580 and SP600125 (specific inhibitors of ERK, p38 and JNK, respectively) markedly inhibited the $\mathrm{IKK} / \mathrm{I} \kappa \mathrm{B} / \mathrm{NF}-\kappa \mathrm{B}$-dependent release of G-CSF, GM-CSF and M-CSF. In conclusion, LNT induces the production of G-CSF, GM-CSF and M-CSF by
\end{abstract}

Correspondence to: Professor Shejiao Dai, Department of Gastroenterology, The Second Affiliated Hospital, Medical School of Xi'an Jiaotong University, 157 XiWu Road, Xi'an, Shaanxi 710004, P.R. China

E-mail: daishejiao@qq.com

Key words: chemotherapy toxicity, bone marrow-derived macrophages, lentinan, colony-stimulating factor, NF- $\kappa \mathrm{B}, \mathrm{MAPK}$ activating the MAPK/NF- $\kappa \mathrm{B}$ signalling pathway in BM cells, thereby mitigating THP-induced myelosuppression.

\section{Introduction}

Therarubicin (THP) is an anticancer agent that acts on the chemical structure of DNA to inhibit the abnormal proliferation of cancer cells. THP is primarily used for the treatment of bladder and ureter cancer (1), malignant lymphoma (2), acute leukaemia (3), breast (4) and ovarian cancer (5). The most common side-effect of THP is bone marrow (BM) suppression (as with most anticancer drugs), which primarily manifests as granulocytopaenia (6). Recombinant human granulocyte colony-stimulating factor (rhG-CSF) is often used to prevent granulocytopaenia after radiotherapy and chemotherapy in the clinic $(7,8)$. rhG-CSF is associated with high treatment costs and adverse reactions (9); therefore, the identification of an effective, safe and inexpensive treatment regimen is imperative. Lentinan (LNT) (Fig. 1A), a glucan extracted from dried shiitake mushrooms (Lentinula edodes), exhibits pharmacological effects such as tumour suppression, immune regulation and antioxidation both in vivo and in vitro (10). It was recently reported that LNT protects against radiation-induced spleen cell damage (11) and alleviates the BM damage induced by paclitaxel (12). However, no study has reported the role of LNT in THP-induced myelosuppression.

Human CSF is mainly secreted by BM cell lines (particularly by $\mathrm{BM}$ macrophages) and includes granulocytic colony-stimulating factor (G-CSF), granulocytic-macrophage colony-stimulating factor (GM-CSF) and macrophage colony-stimulating factor (M-CSF). The secretion of these factors by the BM is inducible. As a common inducer, the strong inflammatory agent lipopolysaccharides (LPSs) activate BM macrophages through multiple signalling pathways, such as MAPK, NF- $\mathrm{BB}$ (13) and PI3K-AKT (14), thereby inducing the production of various inflammatory cytokines (13) and colony-stimulating factors (15). CSF receptors (CSFRs) are located on the surface of granulocyte-committed haematopoietic progenitor, neutrophils and endothelial cells (16). When CSF binds to the receptor, tyrosine phosphorylation of JAK family proteins occurs to recruit STAT family proteins. Once phosphorylated by JAK, STAT enters the nucleus and binds 
to the promoter region of target genes, initiating the expression of effector proteins and inducing the differentiation of haematopoietic progenitor cells into mature granulocytes (17) to mediate the immune and haematopoietic systems. However, the expression of a variety of inflammatory factors induced by LPS may increase the risk of immunosuppression after chemotherapy. Therefore, researchers are actively searching for mild inflammatory immunomodulatory agents to reduce this risk.

In the present study, Swiss mouse cells were cultured in vitro and used as models to determine the effect of LNT on the mitigation of THP-induced myelosuppression. BM-derived macrophages (BMDMs) cultured in vitro were used as a model to examine the mechanism of LNT-induced CSF production.

\section{Materials and methods}

Reagents and animals. THP was purchased from Aladdin (Shanghai, China). LNT was purchased from Kanghaipharm (Nanjing, China). All antibodies were purchased from Cell Signaling Technology (Danvers, MA, USA). Secondary antibodies were purchased from LI-COR Biosciences (Lincoln, $\mathrm{NE}$, USA). Inhibitors of ERK, p38, JNK and NF- $\mathrm{B}$ were purchased from Sigma-Aldrich (St. Louis, MO, USA). Swiss mice were provided by Vital River (Beijing, China). Six-week-old male and female mice weighing $20 \pm 2 \mathrm{~g}$ were used in the experiments. Swiss mouse programs were implemented following a protocol approved by the Institutional Animal Care Committee of Shanghai Institute of Biochemistry and Cell Biology (Shanghai, China).

BMDMs. The hind femur of Swiss mice was collected. After the muscle tissue was removed, BM was obtained by flushing the femur with phosphate-buffered saline (PBS) containing $1 \%$ penicillin/streptomycin. Following filtration and centrifugation, an erythrocyte lysis buffer (Beyotime, Shanghai, China) was added, and the BM was placed in an incubator. $\mathrm{BM}$ cells were collected by centrifugation and resuspended in RPMI-1640 growth medium containing 1\% penicillin/streptomycin, 4\% foetal bovine serum (FBS) and 8\% L929 cell conditioned medium (L929-CM). The medium was exchanged every 3 days for the resuspended BM cells. Cells that exhibited adherent growth were considered BMDMs. When all BM cells were adherent, the medium was changed to BMDM medium containing 10\% FBS and 10\% L929-CM for culture and experiments. Cells were counted using a cell counter (18).

L929-CM: L929 cells were purchased from the American Type Culture Collection (ATCC; Rockville, MD, USA) at 50\% confluency and were seeded in RPMI-1640 medium with $10 \%$ FBS for 5 days. The culture solution was collected and filtered through a $0.22-\mu \mathrm{m}$ membrane filter. The filtrate was stored at $-20^{\circ} \mathrm{C}$ for future usage.

Cell viability assays. Cells were seeded into 96-well plates at $1 \times 10^{4}$ cells/well and cultured for $24 \mathrm{~h}$. After $24 \mathrm{~h}$ of incubation with LNT, the medium was exchanged and $10 \mu \mathrm{l}$ of Cell Counting Kit-8 (CCK-8) reagent (Dojindo, Kumamoto, Japan) was added. The culture was incubated for another $2 \mathrm{~h}$ at $37^{\circ} \mathrm{C}$ before the absorbance was measured at $450 \mathrm{~nm}$ using a microplate reader (Thermo, Waltham, MA, USA).
May-Grïnwald and Giemsa (MGG) staining of blood and $B M$ smears. The total number of cells in the blood and $\mathrm{BM}$ was counted using a haemocytometer. Blood and BM samples were stained with MGG reagents (Sigma-Aldrich). The slides were examined under an inverted microscope at a magnification of $x 100$. The relative number of cells was calculated (19).

Cytokine assays. Blood samples were collected by cardiac puncture. Whole blood was allowed to stand overnight at $4{ }^{\circ} \mathrm{C}$ and was centrifuged at $12,000 \mathrm{rpm}$ for $20 \mathrm{~min}$ to obtain the supernatant. Following drug treatment or transfection, the cultured cells were centrifuged at $12,000 \mathrm{rpm}$ for $5 \mathrm{~min}$. The levels of G-CSF, GM-CSF, M-CSF, TNF- $\alpha$, IL- 6 and IL- $1 \beta$ were assayed using Quantikine ELISA kits developed for the corresponding mouse cytokines (R\&D Systems, Minneapolis, MN, USA).

Confocal laser scanning microscopy analysis of p65 nuclear import. Following treatment with LNT, BMDMs were fixed with $4 \%$ paraformaldehyde and incubated with an anti-p65 antibody overnight. Cell nuclei were stained with 4',6-diamidino-2-phenylindole (DAPI; Sigma-Aldrich) for $20 \mathrm{~min}$. After rinsing with PBS, the nuclear import of p65 was examined using a confocal laser scanning microscope (magnification, x2400) (Olympus, Tokyo, Japan).

MPO activity assays in blood and BM cells. Blood samples were collected by cardiac puncture, and BM samples were obtained by washing the tibiofemoral cavity with PBS. Myeloperoxidase (MPO) activity was assayed using an MPO Colorimetric Activity Assay kit (Sigma-Aldrich) and is expressed as $\mathrm{nmol} / \mathrm{min} / \mathrm{ml}$ (milliunit $/ \mathrm{ml}$ ).

Haematoxylin and eosin (H\&E) staining. Mice were decapitated, and the complete femur was immersed in $4 \%$ paraformaldehyde for $24 \mathrm{~h}$, followed by decalcification with $50 \%$ formic acid and 15\% sodium citrate for $48 \mathrm{~h}$. The standard histological technique of paraffin embedding was used to create longitudinal $5-\mu \mathrm{m}$ sections. The sections were stained with $\mathrm{H} \& \mathrm{E}$ and examined under an inverted microscope (magnification, x40) (IX71; Olympus).

Western blotting. The cells were rinsed with PBS and lysed on ice using a lysis buffer (Beyotime) for $40 \mathrm{~min}$. The cell lysate was boiled with 2 X SDS loading buffer and loaded onto a gel for SDS-PAGE. The gels were transferred to a nylon membrane and blocked with 5\% non-fat milk. The membrane was incubated with a primary antibody at $4^{\circ} \mathrm{C}$ overnight, followed by incubation with a fluorescent secondary antibody for $2 \mathrm{~h}$. Images were collected and analysed using the Odyssey imaging system (LI-COR Biosciences).

Statistical analysis. All data are shown as the mean \pm standard deviation (SD). The results were compared between groups using the t-test and one-way analysis of variance (ANOVA). $\mathrm{P}<0.05$ was considered to indicate a statistically significant result. Statistical analyses were performed with SPSS 19.0 software (SPSS, Inc., Chicago, IL, USA). 
A

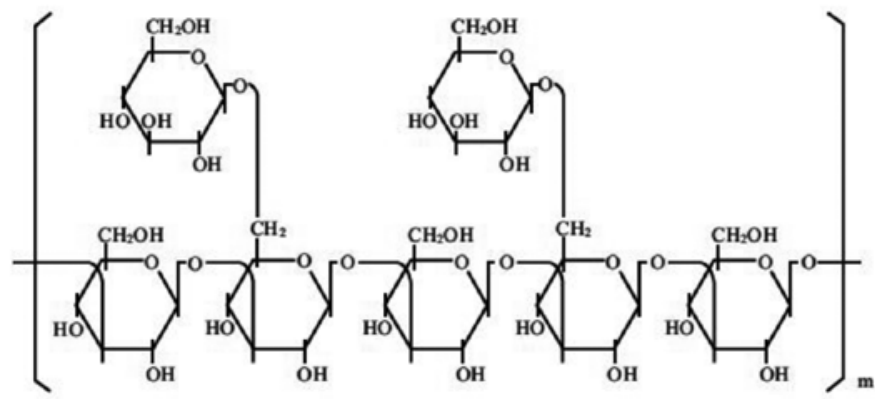

B

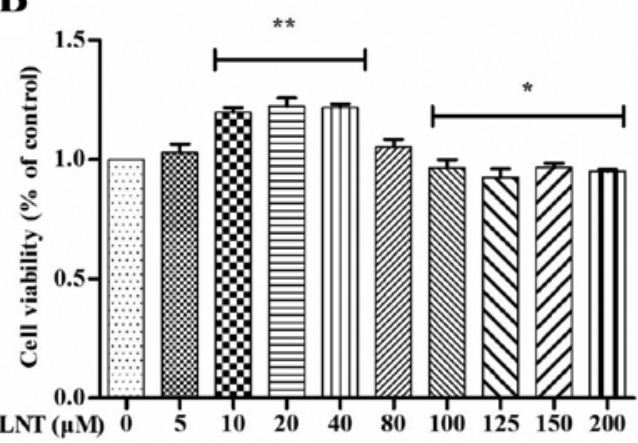

D

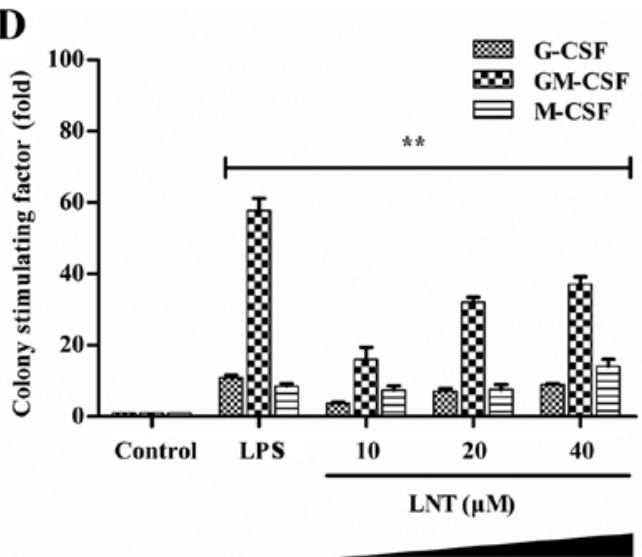

C

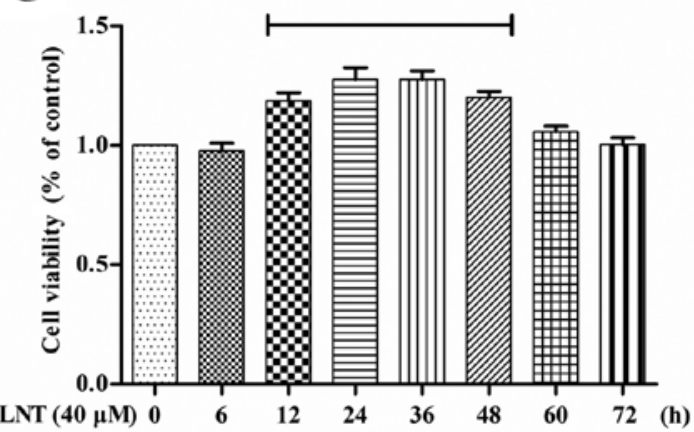

$\mathbf{E}$

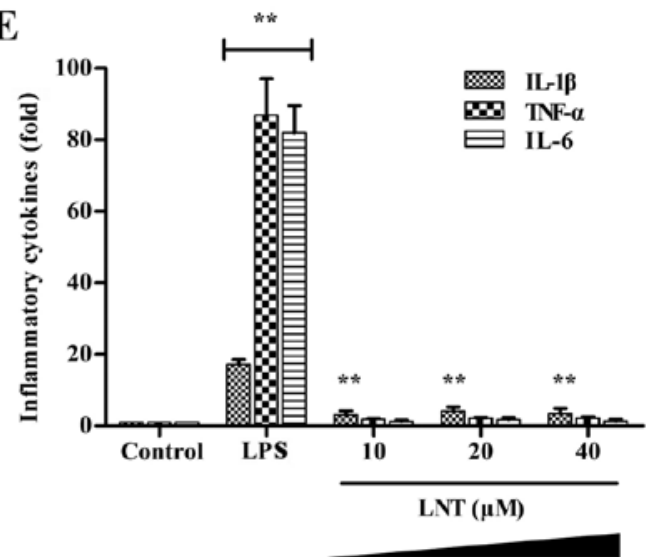

Figure 1. Effect of lentinan (LNT) on colony-stimulating factor and pro-inflammatory cytokine production in BM-derived macrophages (BMDMs). (A) Chemical structure of LNT. (B) BMDMs were incubated with various concentrations of LNT $(0,5,10,20,40,80,100,125,150$ and $200 \mu \mathrm{M})$ for $24 \mathrm{~h}$, and cell viability was then determined by the CCK-8 assay. (C) BMDMs were incubated with $40 \mu \mathrm{M}$ LNT for 0, 6, 12, 24, 36, 48, 60 and $72 \mathrm{~h}$, and cell viability was then determined by the CCK-8 assay. (D and E) BMDMs were incubated with 10, 20 or $40 \mu \mathrm{M} \mathrm{LNT}$ or $200 \mathrm{ng} / \mathrm{ml}$ LPS for $48 \mathrm{~h}$. The secretion of G-CSF, GM-CSF, M-CSF, TNF- $\alpha$, IL- 6 and IL- $1 \beta$ was measured using an ELISA kit. Data are presented as the mean \pm SD of 5 independent experiments; ${ }^{*} \mathrm{P}<0.05$ and ${ }^{* *} \mathrm{P}<0.01$ vs. the control groups.

\section{Results}

Effect of LNT on colony-stimulating factor and pro-inflammatory cytokine production in the BMDMs. To confirm that LNT was not toxic to BMDMs, we incubated BMDMs with various concentrations of LNT $(0,5,10,20,40$, $80,100,125,150$ and $200 \mu \mathrm{M}$ ) for $24 \mathrm{~h}$ and then determined the cell viability by the CCK-8 assay (Fig. 1B). BMDM viability after different periods of incubation $(0,6,12,24$, $36,48,60$ and $72 \mathrm{~h}$ ) with $40 \mu \mathrm{M}$ LNT is shown in Fig. 1C. LNT exerted no toxic side-effects on BMDMs and increased the cell viability over the concentration range of 5-40 $\mu \mathrm{M}$ for 6-48 h. We thus defined 10, 20 and $40 \mu \mathrm{M}$ LNT as low, medium and high doses at the cellular level. To evaluate the inflammatory and immunomodulatory effect of LNT in the
BMDMs, we incubated the cells with 10, 20 and $40 \mu \mathrm{M}$ LNT or $200 \mathrm{ng} / \mathrm{ml}$ LPS as a positive control group for $24 \mathrm{~h}$. Then, we collected the culture supernatant and assessed the secretion of the CSFs G-CSF, GM-CSF and M-CSF (Fig. 1D) and the pro-inflammatory cytokines TNF- $\alpha$, IL- 6 and IL-1 $\beta$ (Fig. 1E) using an ELISA kit. We found that LNT significantly stimulated production of G-CSF, GM-CSF, M-CSF and IL-1 $\beta$ without affecting TNF- $\alpha$ and IL-6 secretion by the BMDMs. The results indicated that LNT significantly stimulated CSF production and induced mild inflammation in the BMDMs.

Effect of LNT on NF- $\kappa B$ signalling in the BMDMs. To determine the signals upstream to the generation of G-CSF, GM-CSF and M-CSF by LNT, we incubated cells with 10, 20 and $40 \mu \mathrm{M}$ LNT for $1 \mathrm{~h}$ and determined the activation of IKK $\alpha / \beta$, I KB $\alpha$ and $\mathrm{p} 65$ 

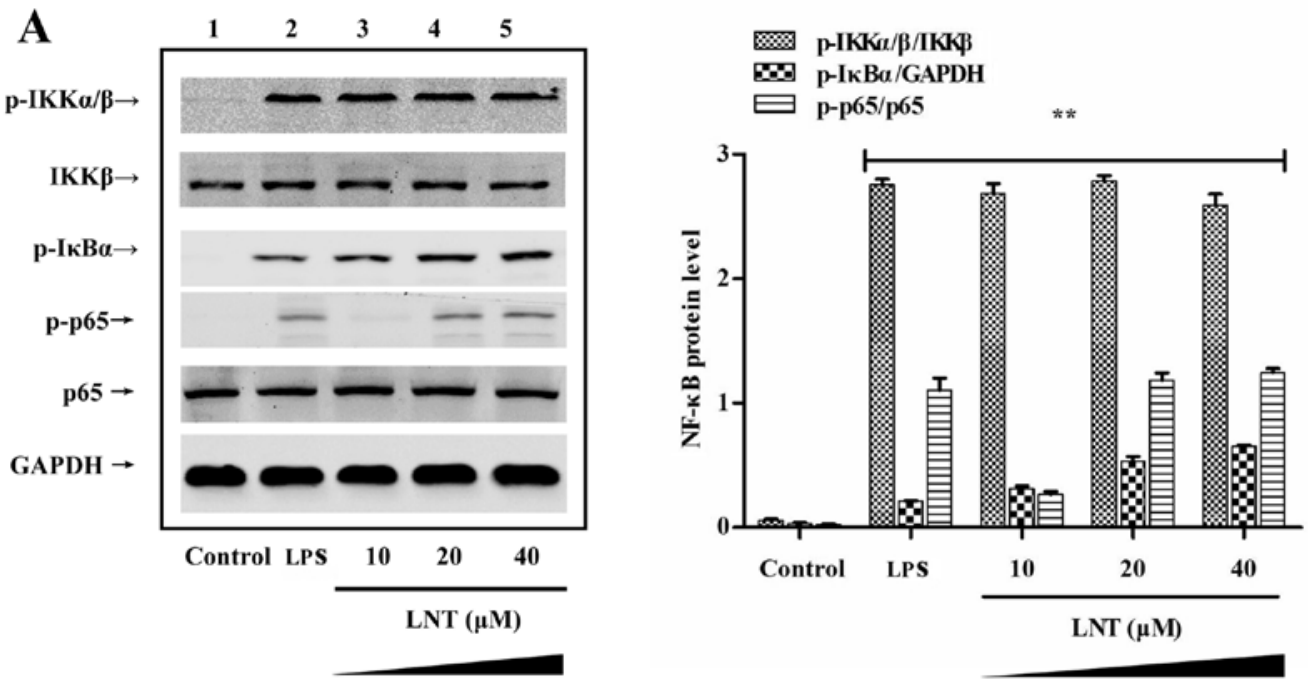

B

DAPI

Anti-p65

Merged

Bright Field

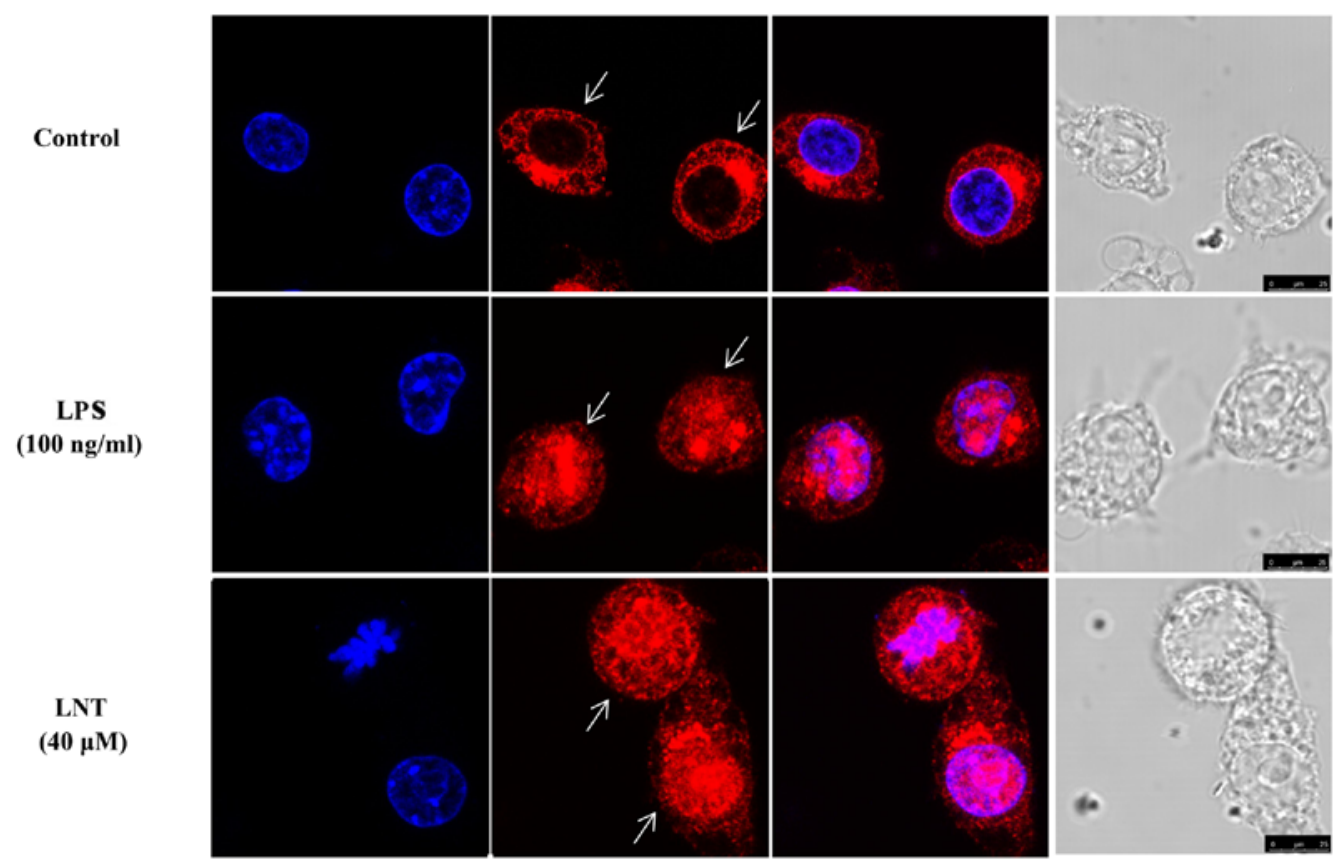

Figure 2. Effect of lentinan (LNT) on NF- $\mathrm{KB}$ signalling in the BM-derived macrophages (BMDMs). (A) BMDMs were incubated with 10, 20 and $40 \mu \mathrm{M}$ LNT for $1 \mathrm{~h}$, and the activation of IKK $\alpha / \beta$, IкB $\alpha$ and p65 was determined by western blotting. (B) BMDMs were incubated with $40 \mu \mathrm{M}$ LNT for $1 \mathrm{~h}$, an anti-p65 antibody was labelled with red fluorescent protein, and the nuclear import of p65 NF- $\mathrm{kB}$ was examined by laser confocal microscopy. Data are presented as the mean $\pm \mathrm{SD}$ of three independent experiments; ${ }^{* *} \mathrm{P}<0.01 \mathrm{vs}$. the control groups.

by western blotting. Additionally, an anti-p65 antibody was labelled with red fluorescent protein, and the nuclear import of p65-NF- $\mathrm{kB}$ was examined by laser confocal microscopy. We found that LNT activated NF- $\kappa \mathrm{B}$ (Fig. 2A) and induced the nuclear import of p65-NF- $\kappa \mathrm{B}$ (Fig. 2B). To examine the relationship between NF- $\mathrm{NB}$ and G-CSF, GM-CSF, M-CSF in our experimental system, we pre-incubated BMDMs for $2 \mathrm{~h}$ with the NF- $\kappa \mathrm{B}$-specific inhibitor BAY 11-7082 $(25 \mu \mathrm{M})$. After $24 \mathrm{~h}$, ELISA assays were performed to examine the effects of the BAY 11-7082 inhibitor on G-CSF, GM-CSF and M-CSF secretion (Fig. 4A-C). The LNT-induced expression of G-CSF, GM-CSF and M-CSF was mediated by the NF- $\kappa \mathrm{B}$ signalling pathway.
Effect of LNT on MAPK signalling in the BMDMs. To determine the signals upstream of LNT-mediated activation of $N F-\kappa B$ signalling, we incubated cells with 10, 20 and $40 \mu \mathrm{M}$ LNT for $30 \mathrm{~min}$ and determined the activation of ERK, JNK and p38 by western blotting. We found that LNT activated the MAPKs ERK, JNK and p38 in a dose-dependent manner (Fig. 3A). To confirm that MAPKs signal upstream of NF- $\kappa \mathrm{B}$-dependent production of G-CSF, GM-CSF and M-CSF in our system, the levels of the signalling proteins IKK $\alpha / \beta$, I $\kappa \mathrm{B} \alpha$ and $\mathrm{p} 65$ were measured after a 1-h treatment with $40 \mu \mathrm{M}$ LNT. BMDMs were pre-treated with the specific ERK inhibitor U0126 $(10 \mu \mathrm{M})$, the specific JNK inhibitor SP600125 $(50 \mu \mathrm{M})$ and the specific p38 inhibitor SB203580 $(20 \mu \mathrm{M})$ for $2 \mathrm{~h}$. As shown in Fig. 3B, 
A

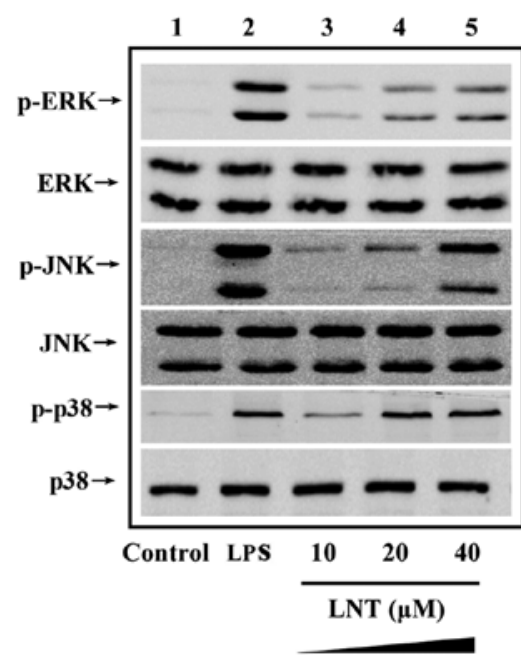

B

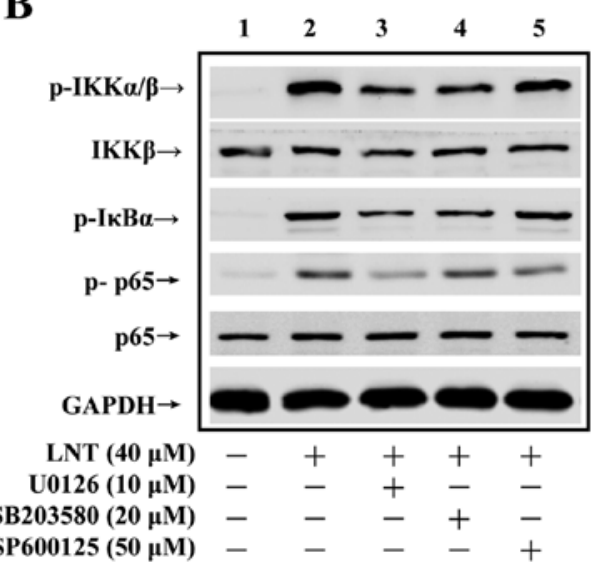

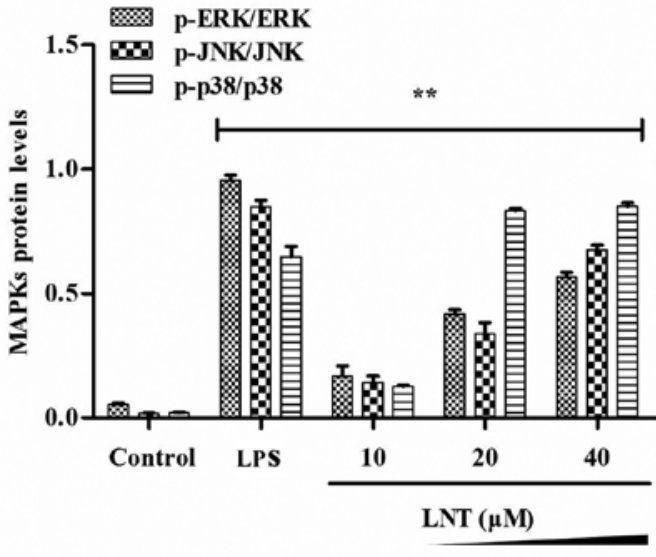

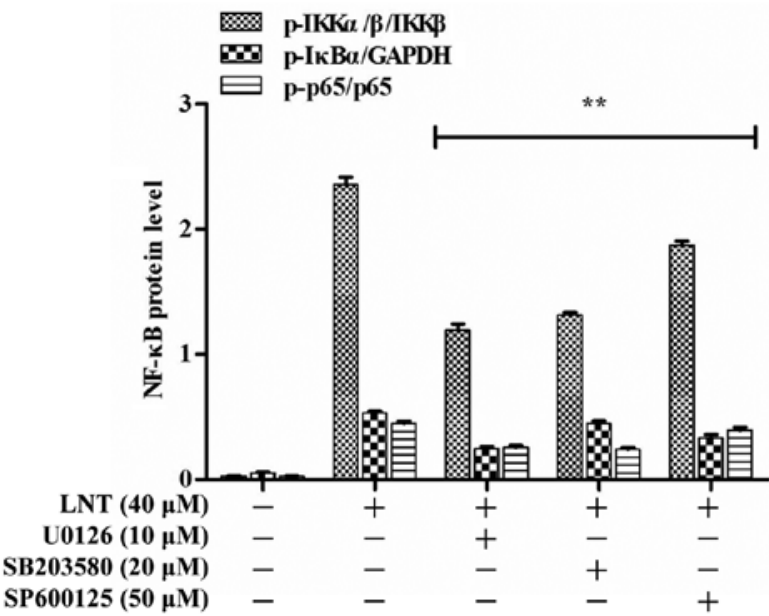

Figure 3. Effect of lentinan (LNT) on MAPK signalling in BM-derived macrophages (BMDMs). (A) BMDMs were incubated with 10, 20 or $40 \mu \mathrm{M}$ LNT for $30 \mathrm{~min}$ and ERK, JNK and $\mathrm{p} 38$ activation was determined by western blotting. BMDMs were pre-treated with $\mathrm{U} 0126(10 \mu \mathrm{M}), \mathrm{SP} 600125(50 \mu \mathrm{M})$ or SB203580 $(20 \mu \mathrm{M})$ for $2 \mathrm{~h}$ and then incubated with $40 \mu \mathrm{M} \mathrm{LNT}$ for $1 \mathrm{~h}$. (B) The activation of IKK $\alpha / \beta$, I $\kappa \mathrm{B} \alpha$ and p65 was determined by western blotting. Data are presented as the mean $\pm \mathrm{SD}$ of three independent experiments; ${ }^{* * *} \mathrm{P}<0.01 \mathrm{vs}$. the control groups.

the suppression of ERK, JNK and p38 phosphorylation also decreased the phosphorylation of IKK $\alpha / \beta$, IкB $\alpha$ and $p 65$. We also measured the effect of U0126, SP600125 and SB203580 on the production of G-CSF, GM-CSF and M-CSF induced by LNT after $24 \mathrm{~h}$. As shown in Fig. 4A-C, the suppression of MAPKs decreased NF- $\kappa B$-dependent G-CSF, GM-CSF and M-CSF production. These results indicate that LNT increased NF- $\mathrm{KB}$ activation by activating the ERK, JNK and p38 signalling proteins, which in turn upregulated G-CSF, GM-CSF and M-CSF production.

Effect of LNT on serum G-CSF, GM-CSF and M-CSF levels in mice treated with THP chemotherapy. MPO, a specific marker of myelocytes, is present in the azurophilic granules of cells from the myeloid lineage (mainly neutrophils and monocytes). To evaluate the effect of LNT on THP-induced myelosuppression in Swiss mice, we administered various concentrations of THP (single dose) by tail vein injection and LNT (one dose every $12 \mathrm{~h}$ ) by intraperitoneal injection after the administration of THP. Seventy-two hours later, blood samples were collected by cardiac puncture to measure serum MPO activity. According to the experimental results (Fig. 5A and B), we selected $25 \mathrm{mg} / \mathrm{kg}$ THP as the toxic concentration and
$20 \mathrm{mg} / \mathrm{kg} \mathrm{LNT}$ as the therapeutic concentration at the animal level. Swiss mice $(n=120)$ were then randomly divided into a control group (10 mice), a THP treatment group (55 mice) and an LNT + THP treatment group (55 mice). Excluding the control group, all of the remaining mice were administered a single tail vein injection of THP $(25 \mathrm{mg} / \mathrm{kg})$, followed by an intraperitoneal injection of LNT $(20 \mathrm{mg} / \mathrm{kg} / 12 \mathrm{~h})$. The animals were decapitated to obtain bilateral femurs after blood sampling by cardiac puncture at $1,3,5,7,9,11,13,15,17,19$ and 21 days. Similarly to the in vitro results, LNT increased the levels of G-CSF, M-CSF and GM-CSF in the serum of the mice on days 3,9 and 3 after THP administration, respectively (Fig. 5C-E). These data demonstrated that LNT drove a marked increase in the serum levels of G-CSF, GM-CSF and M-CSF in mice treated with THP chemotherapy.

Effect of LNT on granulocyte and leukopenia in mice treated with THP chemotherapy. As shown in Fig. 6B-E, we evaluated the numbers of leukocytes and granulocytes in the blood and BM. In the THP treatment group, the numbers of leukocytes and granulocytes were markedly reduced at the early stage of chemotherapy but returned to normal at 21 days. Compared with mice treated with THP, the LNT treatment significantly 
A

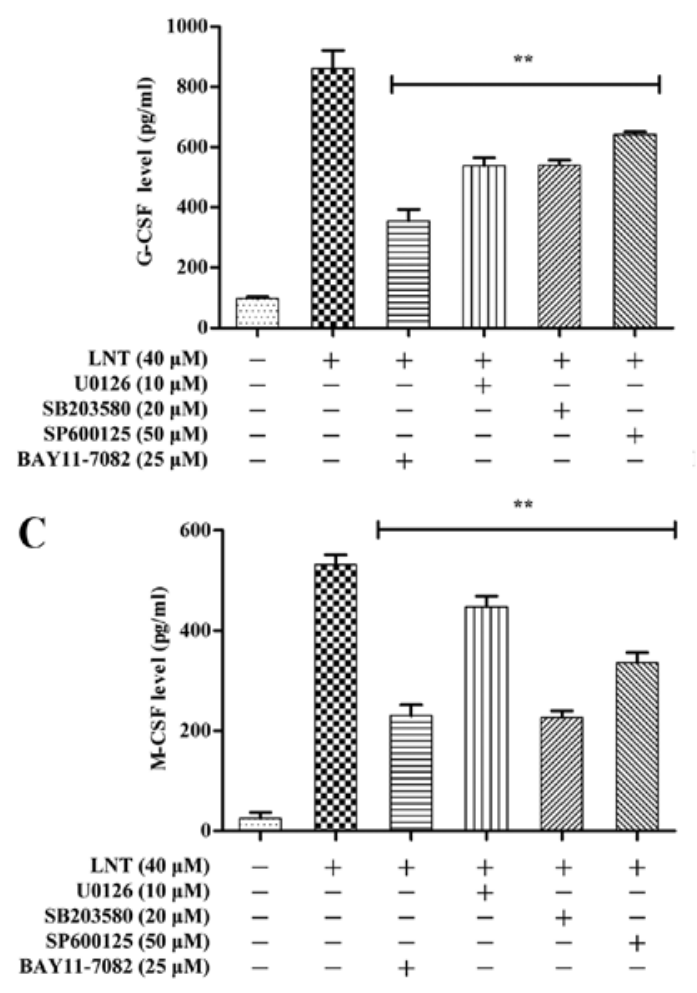

$\mathbf{A}$

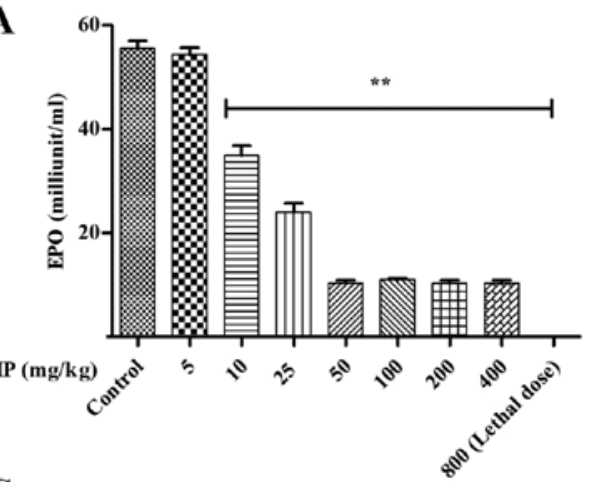

C

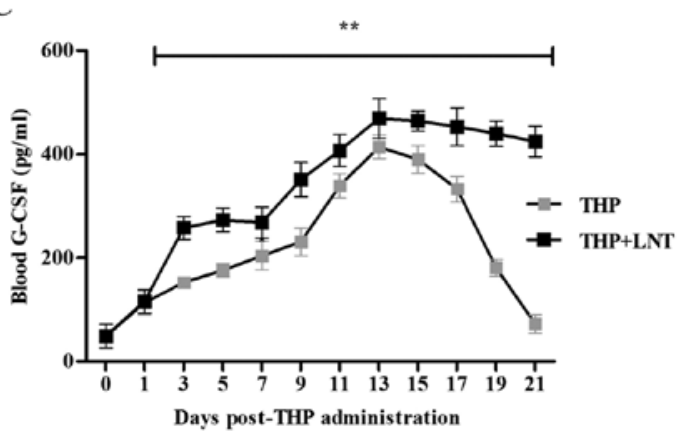

E

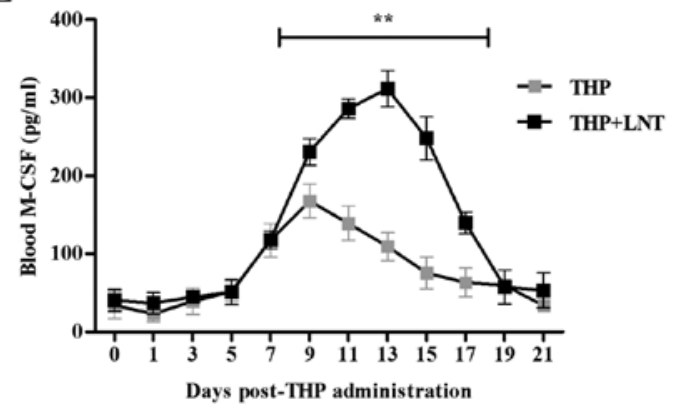

B

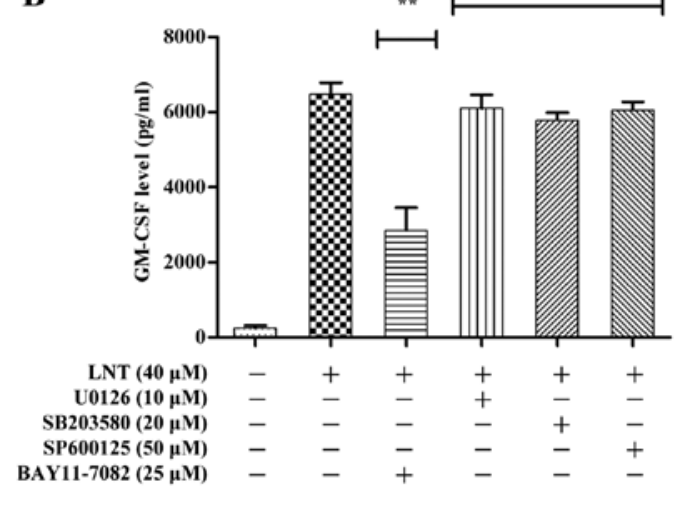

Figure 4. Effect of NF-kB and MAPK inhibitors on G-CSF, GM-CSF, M-CSF in BM-derived macrophages (BMDMs). BMDMs were pre-incubated with the NF- $\mathrm{BB}$-specific inhibitor BAY 11-7082 and MAPK-specific inhibitors for $2 \mathrm{~h}$, and then incubated with $40 \mu \mathrm{M}$ lentinan (LNT) for $48 \mathrm{~h}$. ELISAs were conducted to examine the effects of this inhibitor on (A) G-CSF, (B) GM-CSF and (C) M-CSF secretion. Data are presented as the mean \pm SD of 5 independent experiments; ${ }^{*} \mathrm{P}<0.05$ and ${ }^{* *} \mathrm{P}<0.01$ vs. the control or LNT groups.
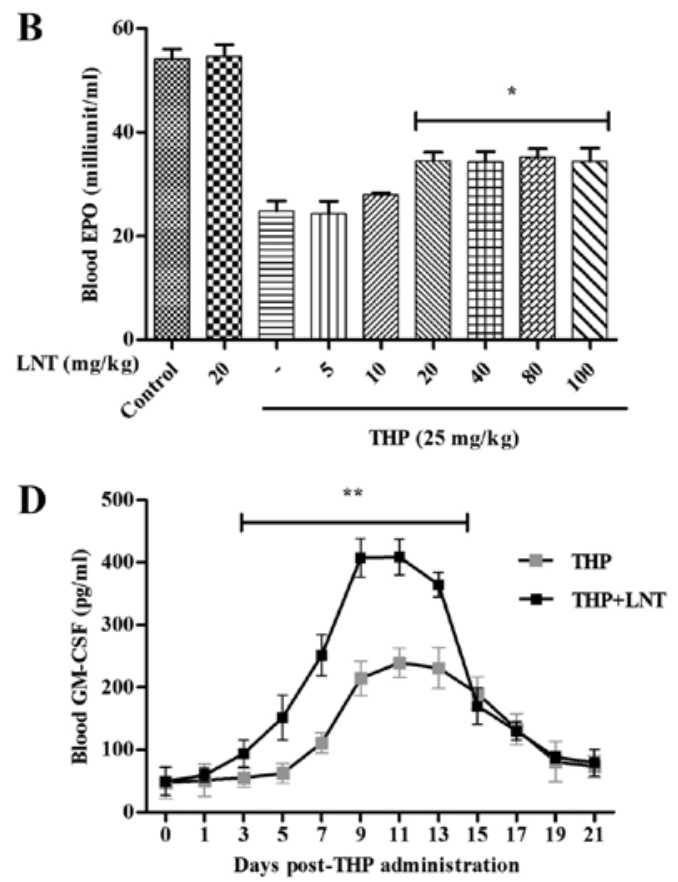

Figure 5. Effect of lentinan (LNT) on serum MPO and G-CSF, GM-CSF, M-CSF levels in mice treated with therarubicin (THP) chemotherapy. Swiss mice were treated with different concentrations of THP by tail vein injection and LNT by intraperitoneal injection after the administration of THP. (A and B) Seventy-two hours later, blood samples were collected by cardiac puncture to measure serum myeloperoxidase (MPO) activity. A total of 120 Swiss mice were randomly divided into a control group, a THP treatment group and an LNT treatment group. Excluding the control group, all of the remaining mice were administered a single tail vein injection of THP, followed by an intraperitoneal injection of LNT. The animals were decapitated to obtain blood sampling by cardiac puncture at 1,3,5,7,9,11,13,15, 17, 19 and 21 days. The levels of blood (C) G-CSF, (D) GM-CSF and (E) M-CSF were measured using ELISA kits. Data are presented as the mean \pm SD of 5 independent experiments; ${ }^{*} \mathrm{P}<0.05$ and ${ }^{* *} \mathrm{P}<0.01$ vs. the monotherapy THP group. 
A
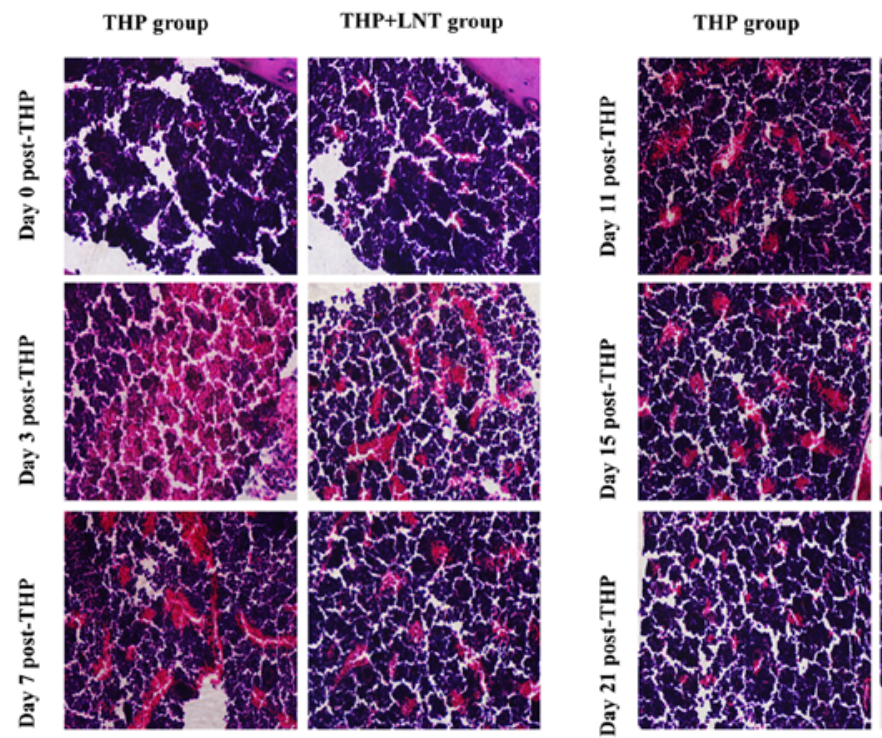

THP+LNT group
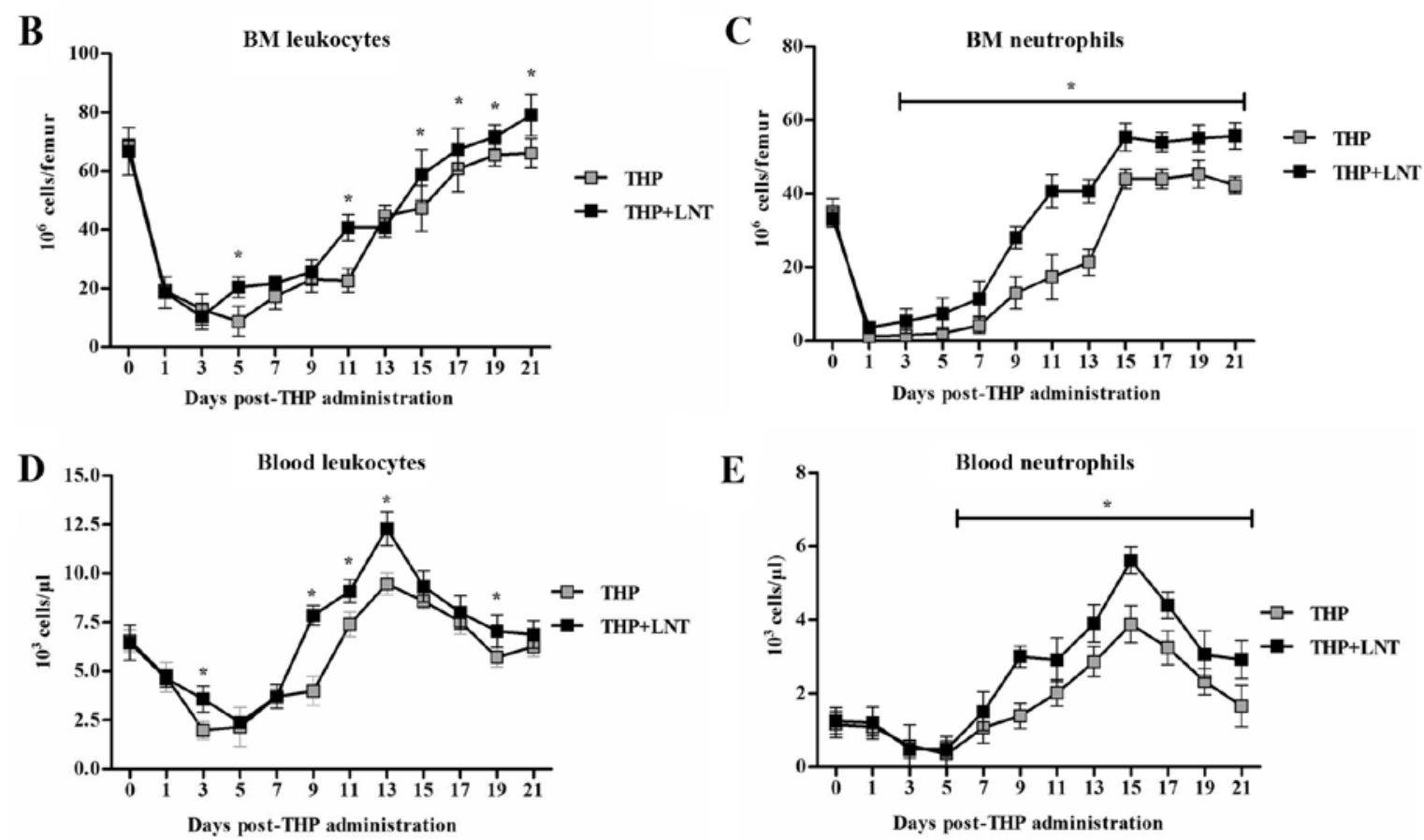

Figure 6. Effect of lentinan (LNT) on therarubicin (THP)-induced acute BM injury, granulocytes and leukopenia in mice. (A) The animals were sacrificed to obtain bilateral femurs, and the BM structure was stained using H\&E and examined with a light microscope (magnification, $x 400$ ). (B-E) The numbers of leukocytes and granulocytes in the blood and BM were evaluated by MGG staining. Data are presented as the mean \pm SD of 5 independent experiments; ${ }^{*} \mathrm{P}<0.05$ vs. the monotherapy THP group.

reduced the recovery period and maintained a higher number of leukocytes and granulocytes. Additionally, H\&E staining of the BM (Fig. 6A) revealed that in the THP treatment group, the BM myeloid/erythroid ratio was markedly reduced at the early stage of chemotherapy; acute BM injury began to heal at 11 days, and complete recovery was achieved at 21 days. The LNT treatment significantly mitigated THP-induced early acute BM injury and reduced the healing period to 7 days, with complete recovery at 15 days.

\section{Discussion}

Therarubicin (THP) is an anthraquinone anticancer drug of the adriamycin family. This agent inserts into the DNA and interacts with topoisomerase II, thereby blocking DNA replication and arresting tumour cells in the G2 phase. As a consequence, the tumour cells cannot enter the cell division phase, which leads to cell death (20). THP inhibits tumour cells with abnormal proliferation and neutrophils with rapid cell division. Granulocytopaenia resulting from THP chemotherapy often suppresses the immune system, increases the risk of infection, extends the treatment period and may be fatal (21). Combination therapy with rhG-CSF is commonly used to mitigate THP-induced granulocytopaenia in the clinic. However, rhG-CSF combination therapy is expensive and associated with multiple side-effects (22). Therefore, new strategies to prevent or reduce the toxicity of chemotherapy at the level of haematopoiesis are essential to improve therapeutic outcome 


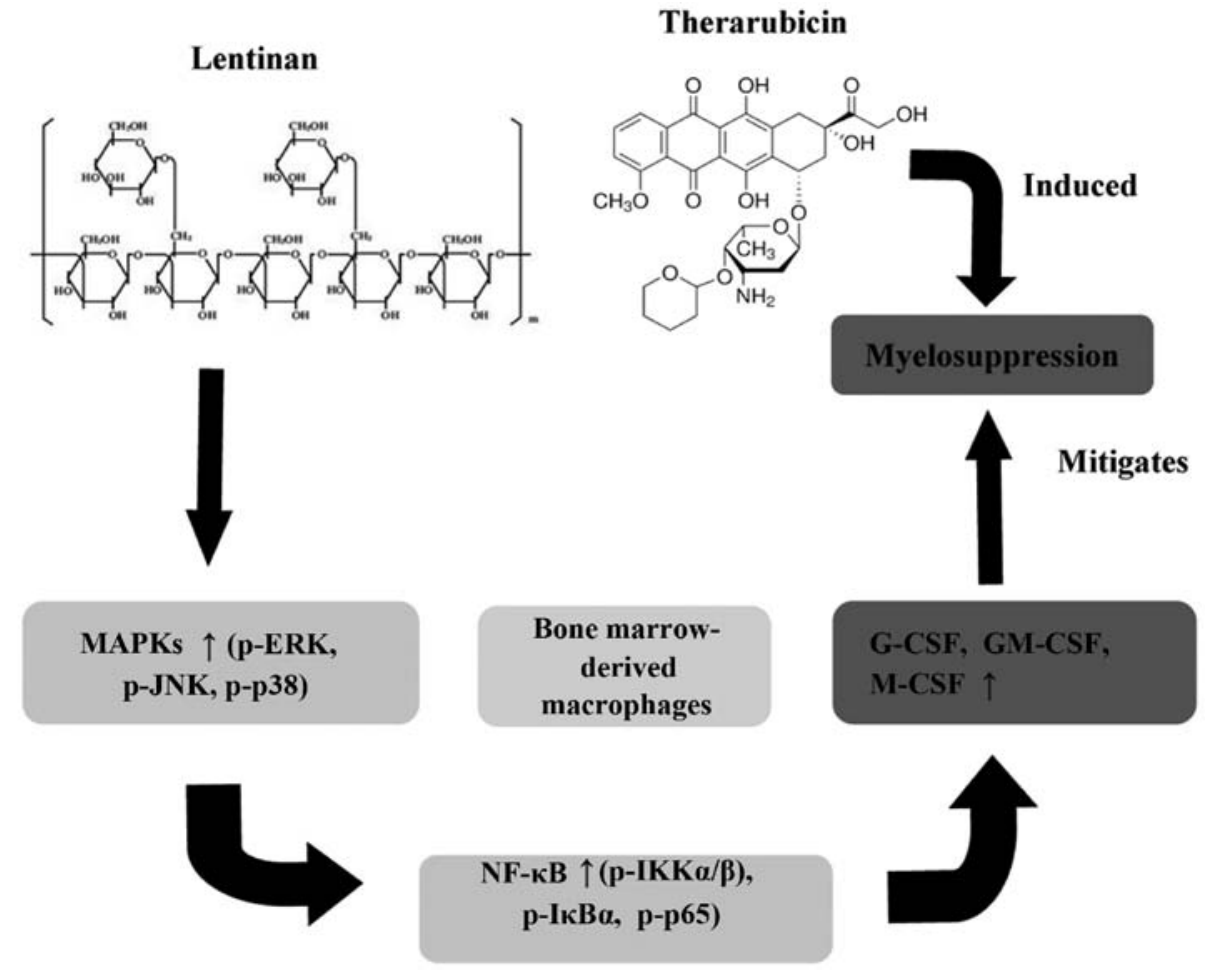

Figure 7. The mechanism by which LNT mitigates THP-induced myelosuppression.

and prognosis for these patients (19). LNT is a $\beta$-D-glucan extracted from shiitake mushrooms (Lentinus edodes). A role for LNT in regulating the immune system has been implicated both in vivo and in vitro by several studies (13-22). Moreover, LNT has antitumour (23), antioxidation (24) and DNA damage repair activities (12). The present study is the first to report that LNT significantly stimulates G-CSF, GM-CSF and M-CSF production in BMDMs and protects against THP-induced myelosuppression in vivo.

Early clinical assessments have demonstrated that the major side-effects of THP include leucopaenia and thrombocytopaenia $(25,26)$; patients cannot undergo the second round of THP treatment until after 21 days $(27,28)$. As expected, we found that $25 \mathrm{mg} / \mathrm{kg}$ THP substantially inhibited blood MPO activity, reduced the numbers of granulocytes and leukocytes in the blood and BM and injured the structure of the BM (ruptured BM capillaries and reduced the myeloid/erythroid ratio) in Swiss mice. This in vivo study demonstrated that LNT significantly mitigated myelosuppression in mice induced by THP injection by regulating the numbers of leukocytes and granulocytes and by suppressing structural injury in the BM. Most importantly, LNT reduced the recovery period of THP-induced myelosuppression to 2 weeks, which suggests that an LNT injection can simultaneously reduce the radiotherapy cycle of THP. This finding has great implications for the treatment of cancer.

After BM transplantation or chemotherapy, an effective means to regulate the immune system is to induce mild inflammation with polysaccharides $(29,30)$. The purpose of our experiments was to identify a polysaccharide with immunomodulatory activity and decreased pro-inflammatory activity to reduce the side-effects of chemotherapy. Many studies have suggested that LNT can be used as an immune modulator to activate the production of various cytokines by macrophages $(10,31)$. In the present study, we isolated BMDMs from BM tissue to examine the mechanism of action of LNT in regulating myelosuppression after chemotherapy. The experiments revealed that non-toxic levels of LNT significantly induced the production of G-CSF, GM-CSF and M-CSF in BMDMs. Further analysis demonstrated that LNT activated the NF- $\kappa \mathrm{B}$ signalling pathway in BMDMs in a dosedependent manner. After LNT-induced BMDMs were treated with NF- $\mathrm{NB}$-specific inhibitors, the production of G-CSF, GM-CSF and M-CSF was significantly inhibited; thus, we believe that the LNT-induced secretion of G-CSF, GM-CSF and M-CSF was due to NF- $\mathrm{B}$ activation. Additionally, the present study showed that LNT stimulated the activation of the ERK, JNK and p38 MAPK signalling pathways in a dosedependent manner. To investigate whether MAPK signalling pathway activation was upstream of $N F-\kappa B$ activation in our experimental system, we used specific MAPK inhibitors that significantly blocked LNT-induced activation of $\mathrm{NF}-\kappa \mathrm{B}$ signalling. More importantly, MAPK inhibitors also blocked LNT-induced G-CSF, GM-CSF and M-CSF production by $\mathrm{BM}$ cells. These results suggest that MAPKs act upstream of $\mathrm{NF}-\kappa \mathrm{B}$ and mediate the production of G-CSF, GM-CSF and M-CSF.

In conclusion, the present study is the first to demonstrate that LNT mitigates myelosuppression caused by THP chemotherapy in vivo. In vitro experiments revealed that LNT stimulated MAPK and NF- $\mathrm{BB}$ signalling to produce G-CSF, GM-CSF and M-CSF in BMDMs (Fig. 7). These experiments provide conceptual and theoretical support for the treatment of granulocytopaenia after clinical chemotherapy. 


\section{References}

1. Ito A, Shintaku I, Satoh M, Ioritani N, Aizawa M, Tochigi T, Kawamura S, Aoki H, Numata I, Takeda A, et al: Prospective randomized phase II trial of a single early intravesical instillation of pirarubicin (THP) in the prevention of bladder recurrence after nephroureterectomy for upper urinary tract urothelial carcinoma: The THP Monotherapy Study Group Trial. J Clin Oncol 31: 1422-1427, 2013

2. Tomita N, Kodama F, Tsuyama N, Sakata S, Takeuchi K, Ishibashi D, Koyama S, Ishii Y, Yamamoto W, Takasaki H, et al: Biweekly THP-COP therapy for newly diagnosed peripheral T-cell lymphoma patients. Hematol Oncol 33: 9-14, 2015.

3. Shimomura Y, Baba R, Watanabe A, Horikoshi Y, Asami K, Hyakuna N, Iwai A, Matsushita T, Yamaji K, Hori T, et al, Japanese Childhood Cancer and Leukemia Study Group (JCCLSG): Assessment of late cardiotoxicity of pirarubicin (THP) in children with acute lymphoblastic leukemia. Pediat Blood Cancer 57: 461-466, 2011

4. Li Y, Tang JH, Huang XE and Li CG: Clinical comparison on the safety and efficacy of fluorouracil/pirarubicin/cyclophosphamide (FPC) with fluorouracil/ epirubicin/cyclophosphamide (FEC) as postoperative adjuvant chemotherapy in breast cancer. Asian Pac J Cancer Prev 12: 1795-1798, 2011.

5. du Bois A, Meerpohl HG, Madjar H, Spinner D, Dall P, Pfisterer J and Bauknecht T: phase II study of pirarubicin combined with cisplatin in recurrent ovarian cancer. J Cancer Res Clin Oncol 120: 173-178, 1994.

6. Zhao H, Yao Y, Wang Z, Lin F, Sun Y and Chen P: Therapeutic effect of pirarubicin-based chemotherapy for osteosarcoma patients with lung metastasis. J Chemother 22: 119-124, 2010

7. Hyun SY, Cheong JW, Kim SJ, Min YH, Yang DH, Ahn JS, Lee WS, Ryoo HM, Do YR, Lee HS, et al: High-dose etoposide plus granulocyte colony-stimulating factor as an effective chemomobilization regimen for autologous stem cell transplantation in patients with non-Hodgkin Lymphoma previously treated with CHOP-based chemotherapy: A study from the Consortium for Improving Survival of Lymphoma. Biol Blood Marrow Transplant 20: 73-79, 2014.

8. Asakuma M, Yamamoto M, Wada M, Ryuge S, Katono K, Yokoba M, Fukui T, Takakura A, Otani S, Maki S, et al: Phase I trial of irinotecan and amrubicin with granulocyte colony-stimulating factor support in extensive-stage small-cell lung cancer. Cancer Chemother Pharmacol 69: 1529-1536, 2012.

9. Gudi R, Krishnamurthy M and Pachter BR: Astemizole in the treatment of granulocyte colony-stimulating factor-induced bone pain. Ann Intern Med 123: 236-237, 1995.

10. Chihara G, Hamuro J, Maeda YY, Shiio T, Suga T, Takasuka N and Sasaki T: Antitumor and metastasis-inhibitory activities of lentinan as an immunomodulator: An overview. Cancer Detect Prev Suppl 1: 423-443, 1987.

11. Liu YH, Ma SD, Fu QJ, Zhao LY, Li Y, Wang HQ and Li MC Effect of lentinan on membrane-bound protein expression in splenic lymphocytes under chronic low-dose radiation. Int Immunopharmacol 22: 505-514, 2014.

12. Attia SM, Harisa GI, Abd-Allah AR, Ahmad SF and Bakheet SA: The influence of lentinan on the capacity of repair of DNA damage and apoptosis induced by paclitaxel in mouse bone marrow cells. J Biochem Mol Toxicol 27: 370-377, 2013.

13. Joo SY, Song YA, Park YL, Myung E, Chung CY, Park KJ, Cho SB, Lee WS, Kim HS, Rew JS, et al: Epigallocatechin3-gallate inhibits LPS-induced NF- $\mathrm{KB}$ and MAPK signaling pathways in bone marrow-derived macrophages. Gut Liver 6: 188-196, 2012.

14. Li X,Liu Y, Wang L, Li Z and Ma X: Unfractionated heparin attenuates LPS-induced IL-8 secretion via PI3K/Akt/NF- $\mathrm{KB}$ signaling pathway in human endothelial cells. Immunobiology 220 $399-405,2015$.
15. Mannello F, Ligi D, Canale M and Raffetto JD: Sulodexide downregulates the release of cytokines, chemokines, and leukocyte colony stimulating factors from human macrophages: Role of glycosaminoglycans in inflammatory pathways of chronic venous disease. Curr Vasc Pharmacol 12: 173-185, 2014.

16. Lee KY, Suh BG, Kim JW, Lee W, Kim SY, Kim YY, Lee J, Lim J, Kim M, Kang CS, et al: Varying expression levels of colony stimulating factor receptors in disease states and different leukocytes. Exp Mol Med 32: 210-215, 2000.

17. Kamezaki K, Shimoda K, Numata A, Haro T, Kakumitsu H, Yoshie M, Yamamoto M, Takeda K, Matsuda T, Akira S, et al: Roles of Stat 3 and ERK in G-CSF signaling. Stem Cells 23: 252-263, 2005

18. Davis BK: Isolation, culture, and functional evaluation of bone marrow-derived macrophages. Methods Mol Biol 1031: 27-35, 2013.

19. Salva S, Marranzino G, Villena J, Agüero G and Alvarez S: Probiotic Lactobacillus strains protect against myelosuppression and immunosuppression in cyclophosphamide-treated mice. Int Immunopharmacol 22: 209-221, 2014

20. Zheng SE, Xiong S, Lin F, Qiao GL, Feng T, Shen Z, Min DL, Zhang CL and Yao Y: Pirarubicin inhibits multidrug-resistant osteosarcoma cell proliferation through induction of $\mathrm{G} 2 / \mathrm{M}$ phase cell cycle arrest. Acta Pharmacol Sin 33: 832-838, 2012.

21. Munck JN, Rougier P, Chabot GG, Ramirez LH, Bognel C, Lumbroso J, Herait P, Elias D, Lasser P and Gouyette A: Phase I and pharmacological study of intra-arterial hepatic administration of pirarubicin in patients with advanced hepatic metastases. Eur J Cancer 30A: 289-294, 1994.

22. Baldo BA: Side effects of cytokines approved for therapy. Drug Saf 37: 921-943, 2014

23. Ina $\mathrm{K}$, Kataoka $\mathrm{T}$ and Ando $\mathrm{T}$ : The use of lentinan for treating gastric cancer. Anticancer Agents Med Chem 13: 681-688, 2013.

24. Fehér J, Chihara G, Vallent K, Deák G, Blázovics A, Gergely P and Kaneko Y: Effect of lentinan on superoxide dismutase enzyme activity in vitro. Immunopharmacol Immunotoxicol 11: 55-61, 1989

25. Sridhar KS, Hussein AM, Benedetto P, Ardalan B, Savaraj N and Richman SP: Phase II trial of 4'-0-tetrahydropyranyladriamycin (pirarubicin) in head and neck carcinoma. Cancer 70: 1591-1597, 1992.

26. KleebergUR, Reichel L, Wander HE, Beyer JH, Essers U, Fiebig HH and Edler L: Phase II study of pirarubicin in metastatic breast cancer. Onkologie 13: 175-179, 1990.

27. Rapoport BL and Falkson G: phase II clinical study of pirarubicin in hormone resistant prostate cancer. Invest New Drugs 10: 119-121, 1992.

28. Wang H, Wang M, Chen J, Tang Y, Dou J, Yu J, Xi T and Zhou C: A polysaccharide from Strongylocentrotus nudus eggs protects against myelosuppression and immunosuppression in cyclophosphamide-treated mice. Int Immunopharmacol 11: 1946-1953, 2011.

29 Yu Q, Nie SP, Wang JQ, Liu XZ, Yin PF, Huang DF, Li WJ, Gong DM and Xie MY: Chemoprotective effects of Ganoderma atrum polysaccharide in cyclophosphamide-induced mice. Int $\mathrm{J}$ Biol Macromol 64: 395-401, 2014

30. Bhatia S, Rathee P, Sharma K, Chaugule BB, Kar N and Bera T: Immuno-modulation effect of sulphated polysaccharide (porphyran) from Porphyra vietnamensis. Int J Biol Macromol 57: 50-56, 2013.

31. Xu X, Pan C, Zhang L and Ashida H: Immunomodulatory beta-glucan from Lentinus edodes activates mitogen-activated protein kinases and nuclear factor-kappaB in murine RAW 264.7 macrophages. J Biol Chem 286: 31194-31198, 2011. 